\title{
ANZ Tennis Hot Shots: Desarrollar, nutrir y fomentar la participación
}

\author{
Patrick McInerney, Rob Urquhart, Rebecca McDonald y Mitchell Hewitt
}

Tenis Australiano

RESUMEN

ANZ Tennis Hot Shots es el programa oficial de desarrollo de Tennis Australia diseñado específicamente para la iniciar en el tenis a los escolares de entre 5 y 12 años. En el centro del programa hay una filosofía innovadora y práctica llamada "Aprendizaje por medio del juego". Esto significa que jugar al tenis o versiones modificadas de éste, es clave en todas las sesiones. El uso del material adaptado, incluyendo las canchas más pequeñas y las pelotas con menor presión, asegura que los niños ingresen al tenis en un entorno adecuado para sus edades y niveles de habilidad. Este método hace que el aprendizaje del tenis sea divertido y más fácil, y como resultado, habrá más niños jugando con un mejor nivel.
Palabras clave: desarrollo del tenis, aprender jugando, material adaptado

Recibido: 14 Ene 2016

Aceptado: 27 May 2017

Autor correspondiente:

Patrick Mclnerney, Tenis

Australiano

Correo electrónico:

pmcinerney@tennis.com.au

\section{INTRODUCCIÓN}

Desde el inicio de ANZ Tennis Hot Shots en 2008, el programa se ha centrado en lograr que más niños jueguen al tenis más frecuentemente. El participante de ANZ Tennis Hot Shots debe jugar en cuatro etapas: Azul, Roja, Naranja y Verde (Tabla 1.) Estas etapas se relacionan con la edad, pero no dependen de ella, pues los niños progresan a medida que logran las competencias tácticas y técnicas pertinentes así como las competencias conductuales en los entornos sociales y emocionales relacionadas con el desarrollo del amor por el juego y por el espíritu deportivo.

Para fomentar la participación infantil se definieron cuatro componentes clave en los cuales el entrenador es fundamental, con la excepción del Juego Comunitario. Los entrenadores de Tenis Australia trabajan con el programa ANZ Tennis Hot Shots de varias formas y su filosofía se integra con todo el sendero de la Formación de Entrenadores.

- Escuelas de ANZ Tennis Hot Shots

- Community Play de ANZ Tennis Hot Shots*

- Entrenamiento de ANZ Tennis Hot Shots

- Competición ANZ Tennis Hot Shots
* Community Play es un programa voluntario organizado por los Clubes Regionales que no tienen acceso a un entrenamiento de tenis calificado.

\begin{tabular}{|c|c|c|c|c|}
\hline Etapa & $\begin{array}{c}\text { Edad } \\
\text { (años) }\end{array}$ & $\begin{array}{c}\text { Tamaño de la } \\
\text { cancha }\end{array}$ & Raqueta & Pelota \\
\hline Azul & $3-5$ & $\begin{array}{c}\text { Variable - definida } \\
\text { utilizando líneas } \\
\text { móviles }\end{array}$ & 19 pulg. & $\begin{array}{c}\text { Pelota roja, } \\
\text { pelota de } \\
\text { espuma, } \\
\text { pelotas } \\
\text { blandas } \\
\text { grandes, } \\
\text { balones }\end{array}$ \\
\hline Roja & $5-8$ & $\begin{array}{c}3 m \times 8.23 m \\
\text { progresando a 5.5 } \\
\times 10.97\end{array}$ & 21 pulg. & $\begin{array}{c}\text { Roja 25\% de } \\
\text { presión }\end{array}$ \\
\hline Naranja & $8-10$ & $6.5 m \times 18 m$ & 23 pulg. & $\begin{array}{c}\text { Naranja } 50 \% \\
\text { de presión }\end{array}$ \\
\hline Verde & $9-12$ & Cancha total & 25 pulg. & $\begin{array}{c}\text { Verde } 75 \% \\
\text { de presión }\end{array}$ \\
\hline
\end{tabular}

Tabla 1. Etapas de ANZ Tennis Hot Shots

PROGRAMA DE ENTRENAMIENTO DE ANZ TENNIS HOT SHOTS

El programa ANZ Tennis Hot Shots proporciona a los entrenadores la capacidad de modificar el juego, adaptando el área de juego (es decir, con canchas más pequeñas o más grandes), cambiando el material (utilizando pelotas con 
diferente presión y raquetas de diferentes tamaños) y cambiando las reglas (es decir, permitiendo el doble bote) teniendo en cuenta el nivel de desarrollo de los niños.

\section{Demográfica de entrenadores}

Actualmente hay 973 entrenadores registrados en ANZ Tennis Hot Shots en Australia. De éstos, el $83 \%$ son hombres y sólo el $17 \%$ son mujeres, lo cual es consistente en toda nuestra fuerza laboral. En lo que respecta a las calificaciones de los entrenadores, el $30 \%$ tiene una calificación de Desarrollo Juniors (equivalente al Entrenador de jugadores iniciantes e intermedios de la ITF) y el $70 \%$ tiene una de Profesional de Club (equivalente a Entrenador de jugadores avanzados de la ITF) o superior.

\section{Beneficios para los entrenadores}

Los entrenadores de ANZ Tennis Hot Shots reciben numerosos beneficios para apoyar el trabajo del programa en las áreas de formación, marketing, material, beneficios para el participante, las experiencias que el dinero no puede comprar y la oportunidad de vincularse con los programas de Tenis para Colegios de Primaria además de con el programa para el Juego de Partidos de ANZ Tennis Hot Shots. Cuando los entrenadores se inscriben, reciben un material inicial que incluye pelotas, redes, y señalización, además de otros recursos generales como certificados, plantillas para marketing, informes sobre la competencia, y acceso a la aplicación de planificación de las lecciones de ANZ Tennis Hot Shots.

La aplicación de entrenamiento de ANZ Tennis Hot Shots se lanzó en 2015 y está disponible globalmente en las tiendas de iTunes y Android. Es una herramienta de fácil uso que contiene más de 120 actividades con grabaciones en video desglosadas en cuatro etapas, para que los entrenadores sigan las necesidades de desarrollo de los niños. Además, proporciona planificación a los entrenadores para que puedan crear sus propias actividades y según sus propios tiempos. Hasta el presente, la aplicación ha sido descargada más de 3500 veces y es una herramienta fantástica para mejorar las habilidades de los entrenadores a lo largo y a lo ancho de Australia.

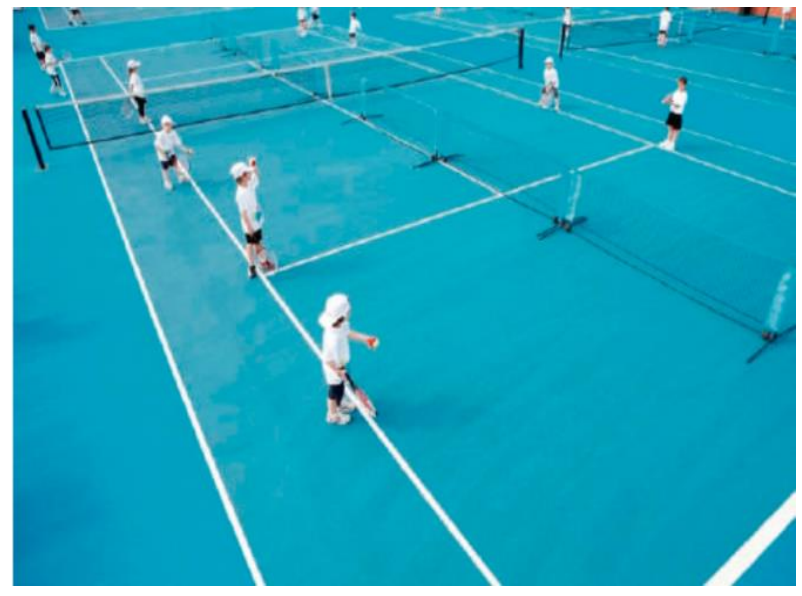

Como recompensa por su significativa contribución, los entrenadores reciben $\$ 2$ para gastar en material por cada niño que inscriben en el programa anualmente. Los entrenadores también pueden solicitar financiación adicional para pintar las líneas o para la instalación de mini-canchas permanentes.

\section{Apoyo de marketing}

Desde el inicio de ANZ Tennis Hot Shots, Tennis Australia ha invertido significativamente en la marca y en el marketing del programa. Aparte de entregar a los entrenadores plantillas de marketing del área local, el programa realiza una campaña nacional de marketing durante la primavera y el verano. Estas campañas que impulsan la adquisición de nuevos negocios para entrenadores han evolucionado desde el inicio del programa y actualmente se centran en demostrar que el tenis es un deporte para toda la vida. La campaña incluye más de 200.000 buscadores únicos para hotshots.tennis.com. au anualmente y captura los datos cuando los padres completan el formulario de expresión de interés, proporcionado la siguiente información relacionada con los nuevos participantes:

Género: $58 \%$ niños y $42 \%$ niñas

Etapa: Azul 18\%, Rojo 48\%, Naranja 21\% y Verde 13\%

Día en el que quieren jugar: Lunes, Martes, Miércoles y Jueves 14\%, Viernes 13\%, Sábado 18\% y Domingo 13\%

Esta información demuestra la importancia de realizar un programa de calidad para niños, puesto que el $66 \%$ de los participantes que se inicia en ANZ Tennis Hot Shots lo hace en las etapas Azul y Roja. También destaca el deseo de los padres de que sus hijos aprendan a jugar al tenis los fines de semana, el $31 \%$ de los pedidos de sesiones son para esos dias. A nivel nacional, hay 654 entrenadores que utilizan la página de internet de ANZ Tennis Hot Shots para promocionar sus lugares de tenis, cada entrenador cuenta como promedio con tres entrenadores de ANZ Tennis Hot Shots.

\section{EL PROGRAMA TENIS PARA COLEGIOS}

El programa Tenis para Colegios Primarios proporciona oportunidades para que maestros y entrenadores pongan en práctica el ANZ Tennis Hot Shots (ANZTHS) en el entorno de los colegios. Los maestros y entrenadores tienen la posibilidad de participar de uno o dos programas, el Programa de Asociación de Colegios, en el cual el tenis forma parte del curriculum de salud y educación física, o del Programa de Juego en el Colegio, que ayuda a los alumnos a pasar de aprender el juego a jugar el juego. Ambos programas apoyan el camino continuo de conectar los colegios con los clubes de la comunidad para lograr una participación duradera.

El Programa de Asociación de Colegios es el programa escolar emblema de Tennis Australia con más de 2.000 colegios entregando tenis en todo el territorio de Australia. El recurso Tenis para Colegios primarios que apoya la entrega de este programa fue desarrollado para alinear con el Curriculum 
Australiano: Salud y Educación Física (AC:HPE, por sus siglas en inglés), y es respaldado por el Consejo Australiano para la Educación física de la Salud y la Recreación (ACHPER, por sus siglas en inglés).

Este recurso cuenta con dos facetas, la Salud Personal Social y de la Comunicad, (PSCH) y Movimiento y Actividad Física (MPA) incorporadas en AC:HPE y sustentadas por el Enfoque de Juego (GSA). Este enfoque formativo hacia la pedagogía, que se aplica en todo el recurso, es importante para el desarrollo de las capacidades generales en el Curriculum Australiano, y por lo tanto, se puede utilizar a lo largo de todas las áreas de aprendizaje.

\section{COMPETICIÓN DE ANZ TENNIS HOT SHOTS}

La competición de ANZ Tennis Hot Shots está diseñado para que los niños que ya están aprendiendo, tengan la oportunidad de hacer la transición del aprendizaje del juego al juego de partidos en un entorno amigable y divertido en base a equipos. La idea es proporcionar a los niños una experiencia positiva y fomentar el amor por el juego, a fin de lograr una participación en el tenis para toda la vida. Este programa se apoya con recursos complementarios para lograr una experiencia de juego de calidad. Actualmente 381 centros llevan a cabo el programa de Juego de Partidos en toda Australia. Los tenistas australianos Daria Gavrilova y Thanasi Kokkinakis son los recientes embajadores del programa.

\section{LA PROMOCIÓN DE ANZ TENNIS HOT SHOTS DURANTE EL ABIERTO DE AUSTRALIA Y LOS EVENTOS DE LA OPEN SERIES}

El Abierto de Australia y los eventos de la Serie del Abierto de Australia proporcionan una plataforma para promocionar el programa ANZ Tennis Hot Shots y recompensar a los actuales participantes y organizadores. En el Abierto de Australia las familias y los niños cuentan con muchas oportunidades de tomar una raqueta y jugar. En 2017, más de 35.000 personas visitaron la Zona de Activación de Fans de ANZ Tennis Hot Shots y más de 12.000 niños jugaron en las minicanchas de ANZ Tennis Hot Shots.

Los entrenadores tienen la recompensa de ver a los niños de sus clubes participando de actividades en cancha, antes del inicio de los jpartidos, más de 100 entrenadores y 1.000 niños participaron en 2017. Además, más de 150 jugadores de tenis de ANZ Tennis Hot Shots tuvieron la oportunidad de tirar la moneda para el sorteo en los partidos de individuales del torneo.

En 2017, un record de 17.537 personas asistieron al quinto Día Anual del Tenis Infantil proporcionando a los familias y a sus niños la posibilidad de jugar ANZ Tennis Hot Shots en Melbourne Park el sábado anterior al Abierto de Australia. Este evento familiar incluyó un espectáculo de una hora en la cancha Rod Laver donde juegan los grandes campeones como Novak Djokovic, Roger Federer, Milos Raonic y Daria Gavrilova y quienes se unieron a la Animación de DreamWorks con personajes como Poppy y Branch de la exitosa película Trolls, Los Pingüinos de Madagascar y PO de Kung Fu Panda 3. Estos fueron algunos de los entretenimientos en la cancha central.

\section{CONCLUSIÓN}

El enfoque holístico hacia el desarrollo de ANZ Tennis Hot Shots desde su inicio ha demostrado ser clave para el éxito impulsando una mayor participación en el tenis. El éxito del programa Tenis para Colegios de Primaria ha iniciado a más 380.000 niños a participar de ANZ Tennis Hot Shots en los últimos años. La transición de los niños por el camino de ANZ Tennis Hot Shots al Juego de Partidos proporciona una plataforma para el continuo éxito de la participación en el tenis en Australia para los años futuros.

El rol del entrenador es clave para el continuo desarrollo del programa pues proporciona una oportunidad para la ya mencionada transición de los niños, desde el tenis en los colegios hasta los programas en clubes. Además los entrenadores tienen la habilidad de inspirar una pasión por el deporte en los niños y desarrollar sus competencias técnicas y tácticas fundamentales para jugar al tenis durante toda su vida.

\section{CONTENIDO ITF ACADEMY RECOMENDADO (HAZ CLICK ABAJO)}

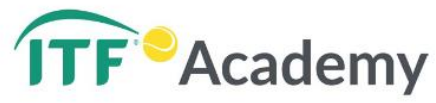

Derechos de Autor (c) 2017 Patrick McInerney, Rob Urquhart, Rebecca McDonald y Mitchell Hewitt.

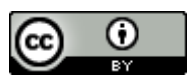

Este texto está protegido por una licencia CreativeCommons 4.0.

Usted es libre para Compartir -copiar y redistribuir el material en cualquier medio o formato- y Adaptar el documento - remezclar, transformar y crear a partir del material- para cualquier propósito, , incluso para fines c omerciales, siempre que cumpla la condición de:

Atribución: Usted debe dar crédito a la obra original de manera adecuada, proporcionar un enlace a la licencia, e indicar si se han realizado cambios. Puede hacerlo en cualquier forma razonable, pero no de forma tal que sugiera que tiene el apoyo del licenciante o lo recibe por el uso que hace de la obra.

Resumendelicencia - Textocompletodelalicencia 